\title{
LA CONCENTRATION MAXIMALE ADMISSIBLE DU PLUTONIUM 239 DANS L'AIR*
}

\author{
J. HAMARD ** \\ (Manuscrit ręu le 28 février 1972)
}

Le plutonium, qui se trouve revêtir actuellement une importance croissante, tant comme combustible nucléaire par son isotope 239 que comme générateur d'énergie par son isotope 238 , se classe également parmi les radioéléments usuels les plus toxiques, et cette toxicité amène à prendre d'exceptionnelles précautions lors de sa manipulation afin de limiter l'exposition des travailleurs à des niveaux acceptables.

Cette radiotoxicité particulièrement élevée du plutonium dépend d'un certain nombre de paramètres dont les principaux sont :

- ses propriétés radioactives: en ce qui concerne le plutonium 239 qui est l'isotope le plus usuel, c'est un émetteur $\alpha$ d'activité spécifique moyenne $\left(6.10^{-2} \mathrm{Ci} / \mathrm{g}\right.$ ) et l'on sait que le rayonnement $\alpha$ possède un transfert linéique d'énergie très élevé dans les tissus (140 keV/ $\mu$ dans l'eau).

- son mode de pénétration dans l'organisme: les modes de contamination les plus fréquents en ce qui concerne les travailleurs sont la contamination respiratoire et la contamination par blessure. On sait en effet que la contamination digestive est peu fréquente et que le plutonium traverse difficilement la barrière digestive (quelques dix millièmes de la quantité ingérée).

- ses propriétés physiques et physico-cbimiques: une caractéristique, dont nous verrons plus loin l'importance, est la granulométrie des aérosols contaminants. Elle intervient lors de l'inhalation en déterminant l'importance du dépôt dans les différents segments de l'arbre respiratoire. Nous verrons par la suite comment on peut tenir compte de ce facteur.

Une autre propriété intéressante est le caractère soluble ou insoluble du composé mis en jeu, ou, pour utiliser une terminologie plus précise, son caractère " transférable ou non transférable " à l'intérieur de l'organisme.

En ce qui concerne le plutonium, celui-ci se rencontre le plus souvent sous deux formes : le nitrate $\mathrm{Pu}\left(\mathrm{NO}_{4}\right)_{3}$ et l'oxyde $\mathrm{Pu} \mathrm{O}$. Le nitrate est

(*) Conférence prononcée à Marcoule le 18 mars 1971 à l'occasion d'une réunion scientifique de la Société Française de Radioprotection.

(**) Département de protection - S.P.S. - Centre d'Etudes Nucléaires. B.P. $\mathrm{n}^{0}$ 6, $9^{2}$ - Fontenay-aux-Roses. 
considéré comme soluble et l'oxyde comme insoluble. En fait, la distinction n'est pas aussi tranchée, car on sait que, lorsqu'il est introduit dans l'organisme sous forme soluble, le plutonium tend à s'insolubiliser et que de même, une faible fraction du plutonium sous forme insoluble subit au niveau du poumon une sorte d'“érosion» qui le solubilise et peut dans une certaine mesure permettre sa pénétration dans l’organisme.

- ses propriétés métaboliques: et en particulier sa vitesse d'élimination à partir d'un site de fixation donné, caractérisé par sa période effective.

Sous forme " non soluble», non transférable, le plutonium va se fixer au niveau du tissu pulmonaire et dans les ganglions lymphatiques pulmonaires. Dans le rapport du Comité II de la C.I.P.R. (publié en 1959) cette période d'élimination était donnée égale à I an. Quant au plutonium " transférable » son métabolisme va l'amener à se fixer sur le squelette qui retient $90 \%$ du plutonium présent dans l'organisme à l'équilibre. Il n'en est éliminé que très lentement : la C.I.P.R., dans son édition de 1959, donne une période biologique dans l'os de 200 ans.

Enfin, un dernier facteur de toxicité est l'énergie effective délivrée au niveau des organes de dépôt. Dans le cas du poumon, cette énergie est de $53 \mathrm{MeV}$ et, dans le cas du squelette, de $270 \mathrm{MeV}$ pour ${ }^{239} \mathrm{Pu}$ (elle est du même ordre de grandeur pour les autres isotopes mis à part $\left.{ }^{241} \mathrm{Pu}\right)$.

\section{CONCENTRATION MAXIMALE ADMISSIBLE DU PLUTONIUM DANS L'AIR}

La radiotoxicité d'un radionucléide se traduit assez bien dans la valeur de sa concentration maximale admissible (CMA). On sait que, dans le cas du plutonium, la quantité maximale admissible (QMA) dans l'organisme, relative au squelette, a été déduite des valeurs obtenues expérimentalement pour le radium 226 (O,I $\mu \mathrm{Ci})$.

Les QMA des deux radionucléides sont dans le rapport inverse des énergies effectives délivrées à l'os :

$$
\begin{gathered}
\frac{\mathrm{QMA}^{239} \mathrm{Pu}}{\mathrm{QMA}^{226} \mathrm{Ra}}=\frac{\text { I } 10}{270} \times \frac{f_{2} \mathrm{Ra}}{f_{2} \mathrm{Pu}} \\
\mathrm{QMA}^{239} \mathrm{Pu}=0, \mathrm{I} \times \frac{\mathrm{II0}}{270} \times \frac{0,99}{0,9}=0,04 \mu \mathrm{Ci} .
\end{gathered}
$$

Si l'on s'en tient à la CMA dans l'air ( $40 \mathrm{~h})$, celle-ci possède deux valeurs distinctes suivant que l'on a affaire à du plutonium " non transférable » pour lequel l'organe de référence est le poumon

$$
\text { 4.10-11 } \mathrm{Ci} / \mathrm{m}^{3}
$$

et sous forme "transférable», pour lequel l'organe de référence est le squelette

$$
\text { 2. } 10^{-12} \mathrm{Ci} / \mathrm{m}^{3} \text {. }
$$


Ces valeurs sont identiques pour la plupart des isotopes du plutonium (seul ${ }^{241} \mathrm{Pu}$ a une CMA moins sévère due à une période plus brève $\left(4,8.10^{3}\right.$ jours) et à une énergie effective plus faible ( $14 \mathrm{MeV}$ seulement au niveau de l'os).

\section{SIGNIFICATION DE LA CMA}

On sait quelle interprétation il convient de donner à cette notion de CMA. C'est une limite, qui, si elle était maintenue en permanence, assurerait le respect des doses maximales admissibles pour les travailleurs. En fait, dans la plupart des cas, il est possible de se maintenir très en dessous de la CMA; mais il est par contre impossible d'empêcher l'occurrence d'un certain nombre d'incidents mineurs entraînant une bouffée de contamination atmosphérique pouvant atteindre des valeurs plusieurs fois supérieures à la CMA, sans qu'il en résulte nécessairement un risque pour les travailleurs.

Aussi considère-t-on la CMA comme une valeur de référence telle que si elle est respectée en moyenne sur un trimestre, les doses maximales admissibles soient elles-mêmes respectées. La concentration d'un radionucléide dans un local peut alors fluctuer entre les limites assez larges puisqu'on peut admettre à la rigueur qu'en travail normal un individu inhale en une fois ce qu'il aurait inhalé en trois mois de travail à la $\mathrm{CMA}_{40 h}$. Ceci revient à substituer pratiquement à la notion de CMA celle de quantité maximale inhalable en un trimestre (avec quelques réserves résultant des différences entre une contamination unique et une contamination chronique).

Cette notion de QMI devient très importante pour l'Ingénieur de Radioprotection car elle est reprise dans les dernières recommandations de la C.I.P.R. et de l'Agence.

\section{IMPORTANCE DU CONTROLE}

Il est évident qu'au cours d'un travail mettant en jeu des aérosols radioactifs, il peut ne pas être possible d'empêcher complètement une émission de radioactivité. Lorsque se produit un tel incident dans lequel sont impliquées des personnes, il est nécessaire de disposer de moyens de contrôle permettant de détecter sitôt que possible cette contamination, de donner l'alarme et de déterminer son importance. En fonction des indications fournies, l'Ingénieur de Radioprotection pourra prendre les mesures qui s'imposent afin de limiter l'exposition des individus. Après l'incident, ces indications serviront à recouper la dosimétrie biologique qui permet d'apprécier l'importance de l'exposition subie par les personnes.

Mais, dans le cas du plutonium 239, cette détection de l'incident est rendue difficile pour deux raisons :

- la valeur très faible de la CMA du ${ }^{239} \mathrm{Pu}$ dans l'air. On sait que, dans un mètre cube d'air, cette activité peut être le fait de quelques grosses particules.

- la présence dans l'atmosphère des produits de filiation émetteurs $\alpha$ du radon et du thoron. 
La toxicité du radon, du thoron et de leurs produits de filiation est plus faible que celle du plutonium : et ceci se traduit dans la valeur de leur CMA dans l'air $3.10^{-7} \mathrm{Ci} / \mathrm{m}^{3}$; mais la concentration de ces radioéléments est loin d'être négligeable. A l'air libre, elle est couramment d'une centaine de $\mathrm{pCi} / \mathrm{m}^{3}$ soit so fois l'activité du ${ }^{239} \mathrm{Pu}$ correspondant à la CMA ( $\mathrm{Pu}$ " transférable, squelette »). On admet que, dans les locaux, cette activité est du même ordre de grandeur, bien que la filtration ait théoriquement pour effet d'éliminer une partie des produits de filiation du radon et du thoron.

Dans le cas du plutonium, on utilisera des appareils dont le rôle principal sera de discriminer l'activité artificielle due aux radiocontaminants émetteurs $\alpha$ de l'activité naturelle, de donner l'alarme en cas de dépassement des niveaux admissibles et d'enregistrer l'importance et l'évolution de la contamination de l'air.

Or la conception de la plupart des appareils existants est telle qu'avant de donner l'alarme un appareil va devoir aspirer une bouffée de contamination et qu'il va donc s'écouler un certain temps, entre le début de la contamination et le déclenchement de l'alarme. Il est donc difficile d'empêcher qu'au cours d'un incident les personnes présentes ne reçoivent une certaine radioexposition.

On estime en général que la sensibilité des appareils actuellement en service correspond à 5 à io $\mathrm{CMA} \times$ heures. Or il est facile à partir de cette expression de l'“exposition» de l'individu d'obtenir une valeur approchée de l'ordre de grandeur de la dose reçue au moyen d'une relation simple reliant l'activité inhalée à la dose engagée.

Ainsi s'exposer pendant ro minutes à une concentration du ${ }^{239} \mathrm{Pu}$ dans l'air égale à 6 fois la CMA produit une « exposition» de I CMA-heure.

Il en résulte une dose engagée de

$$
\frac{30000}{2000}=15 \text { mrems }
$$

ce qui est peu, si l'on sait que ces is mrems ne seront pas reçus en un temps court mais répartis tout au long de la vie professionnelle de l'individu avec un débit de dose moyen de 0,3 mrem/an.

Les performances des appareils existants seraient donc assez satisfaisantes, si cette activité intégrée par l'appareil était représentative de celle que peut inhaler un individu présent dans le même local. Ce n'est malheureusement pas toujours le cas et l'on sait que l'activité inhalée par les personnes présentes peut différer d'un facteur dix de celle inhalée par l'appareil lorsque celui-ci donne l'alarme.

On peut donc souhaiter voir abaisser ce seuil par l'amélioration des appareils de détection, ce à quoi s'attachent les Ingénieurs de Radioprotection. Il est aussi possible de rechercher plus de précision dans l'évaluation des paramètres de l'exposition et de l'évaluation du risque pour les travailleurs.

Or la valeur actuelle de la CMA du plutonium a été établie suivant ces principes de calcul et les schémas de dépôt établis en 1959 par le Comité II de la C.I.P.R. (fig. no I). D'après ce schéma, $12,5 \%$ de la quantité de plutonium "insoluble» inhalée demeurent dans les poumons et en sont éliminés avec une période de $\mathrm{I}$ an; de même, $20 \%$ du plutonium " soluble » inhalé se retrouveraient dans l'os. 


\section{DONNÉES RÉCENTES \\ APPLICATION DU MODĖLE RESPIRATOIRE DE MORROW}

Il peut être intéressant de réexaminer ces valeurs à la lumière de travaux plus récents, dont en particulier le rapport du Task Group on Lungs dynamics de la C.I.P.R. publié en 1966.

Dans cette étude, on substitue, au modèle simplifié de la C.I.P.R. II, une division du tractus-respiratoire en trois compartiments (fig. $\mathrm{n}^{0} 2$ ) :

- le nasopharynx,

- le segment trachéo-bronchique,

- le poumon proprement dit.

Une des hypothèses de travail est que le dépôt dans les différents segments de l'arbre respiratoire est fonction de la granulométrie des particules et du volume d'air inhalé. L'élimination seule dépend de la forme physico-chimique des composés inhalés qui sont divisés en trois classes :

- faible rétention : classe $\mathrm{D}$,

- rétention moyenne : classe $\mathrm{W}$,

- rétention forte : classe Y.

Du point de vue granulométrie, l'aérosol inhalé est supposé avoir une distribution log-normale. Par des méthodes conventionnelles d'échantillonnage, on peut obtenir le diamètre médian en nombre de particules (ou fréquence) ou le diamètre massique médian ainsi que la déviation géométrique standard $\sigma$. Le diamètre massique médian sera le paramètre représentatif de la déposition du nuage de particules dans l'arbre respiratoire.

En se basant sur un certain nombre de travaux théoriques et expérimentaux, le Task Group on lungs dynamics a calculé, pour un certain nombre de diamètres de particules et trois volumes respiratoires $750 \mathrm{~cm}^{3}, 1450 \mathrm{~cm}^{3}, 2150 \mathrm{~cm}^{3}$, le dépôt dans les trois segments de l'arbre respiratoire.

Les courbes de la figure 3 indiquent la relation existant entre le diamètre massique médian en abscisses et en ordonnées les fractions correspondantes de l'activité inhalée déposées dans un compartiment donné pour un volume respiratoire de $1450 \mathrm{~cm}^{3}$. Sur cette figure, chacune des aires hachurées indique la variabilité du dépôt pour un diamètre massique médian, quand le paramètre de distribution varie de 1,2 à 4,5 . Ces courbes méritent qu'on s'y arrête quelques instants. On notera en particulier :

Io l'importance du nasopharynx comme site de dépôt. On voit en effet que 90 à $100 \%$ des particules inhalables d'un D.M.M. compris entre roo et ro $\mu \mathrm{m}$ se dépose dans le nez;

$2^{\circ}$ le dépôt dans le tractus bronchique ne dépend pas du M.M.D. et représente $8 \%$ de l'aérosol inhalé (entre o,os et $100 \mu \mathrm{m}$ ).

$3^{\circ}$ en ce qui concerne le compartiment pulmonaire proprement dit, on observe un dépôt de $25 \%$ qui est celui utilisé dans la C.I.P.R. Il correspond à une granulométrie moyenne très voisine de $\mathrm{I} \mu \mathrm{m}$. On voit aussi que ce pourcentage peut s'élever à $60 \%$ pour un D.M.M. de $0,01 \mu \mathrm{m}$ ou s'abaisser à I $\%$ pour une granulométrie de $100 \mu \mathrm{m}$.

vOL. $7-\mathrm{N}_{2} 2$ 
Dans le schéma proposé par Morrow la fraction de la quantité déposée dans le compartiment pulmonaire et qui y demeure pour un temps long n'est plus de $12 \%$ mais de $18 \%$. De même la période d'élimination à partir du poumon qui était antérieurement de $\mathrm{I}$ an pour le plutonium et de I 20 jours pour les autres radioéléments est susceptible de varier à présent avec la nature physicochimique de l'élément.

On saisit l'intérêt pratique de ces considérations en radioprotection. Il sera intéressant en particulier de disposer d'appareils susceptibles de donner des renseignements précis sur la granulométrie des particules. On pourrait alors distinguer dans un aérosol quelconque une fraction inhalable, d'une fraction constituée par les particules les plus grosses qui ne le seront que très peu.

Ainsi, aux Etats-Unis, Ferran et Hyatr à Los Alamos se sont efforcés de déterminer la fraction "respirable» d'aérosols de plutonium et d'uranium en entendant par là la fraction qui parvient au compartiment “ poumon » de MoRRow. Ils utilisent pour cela un appareil de prélèvement d'air à deux étages, le premier étant constitué d'un cyclone et le second par une membrane filtrante. Pour un aérosol de D.M.M. de $64,5 \mu \mathrm{m}$ ils vérifient que $2 \%$ seulement parviennent au second étage de leur appareil. De même, avec un aérosol de D.M.M. compris entre 0,2 et $0,55 \mu \mathrm{m}, 75$ à $100 \%$ des particules parviennent sur le second étage de l'appareil.

\section{CONCLUSION}

Compte tenu de ces données, que peut-on prévoir de l'évolution des valeurs de la C.M.A. du plutonium dans l'air?

En ce qui concerne la C.I.P.R., celle-ci au vu des conclusions du Groupe de travail de Morrow tiendra compte, dans sa détermination de la valeur de la CMA, de la granulométrie ainsi que de la forme physico-chimique du composé considéré. Ceci conduit au choix d'une CMA correspondant à une granulométrie de référence ainsi qu'au remplacement des actuelles formes solubles et insolubles par trois classes en fonction de la vitesse d'élimination du composé considéré :

- Une classe D (jour) pour les composés à vitesse d'élimination rapide, médiaire,

- Une classe W (semaine) pour les composés à vitesse d'élimination inter-

- Une classe Y (année) pour les composés à vitesse d'élimination lente.

Peut-être pourra-t-on aller plus loin dans la mesure où les études techniques sur les distributions des dimensions des aérosols rencontrés dans les laboratoires fourniraient des résultats suffisamment constants. La CMA pourrait être alors appliquée de façon plus modulée en tenant compte de ces données et en fonction de recommandations actuellement à l'étude à la C.I.P.R. 


\section{DÉFINITIONS RELATIVES A QUELQUES PARAMÈTRES PHYSIQUES}

DiAmètre médian EN MASSE - D.M.M.

Valeur du diamètre des particules, telle que les masses des particules d'un diamètre supérieur ou inférieur soient égales.

DiAMÈTRE MÉdiAN EN NOMBRE DE PARTICULES - D.M.C.

Valeur du diamètre des particules telle que le nombre des particules d'un diamètre supérieur soit égale au nombre des particules d'un diamètre inférieur au diamètre médian.

DiAmÈtre MÉdiAN EN ACTIVITÉ - D.M.A.

Valeur du diamètre des particules telle que l'activité de l'ensemble des particules de diamètre supérieur soit égale à l'activité de l'ensemble des particules de diamètre inférieur.

DIAMÈTRE AÉRODYNAMIQUE.

Diamètre d'une sphère de densité unité ayant la même vitesse de sédimentation que les particules en question.

Si Dr est le diamètre de la sphère de densité unité ayant même vitesse de sédimentation que la particule de diamètre $\mathrm{D}$ de densité $\varphi$, on a :

$$
D_{1}=\varphi \cdot D \text {. }
$$




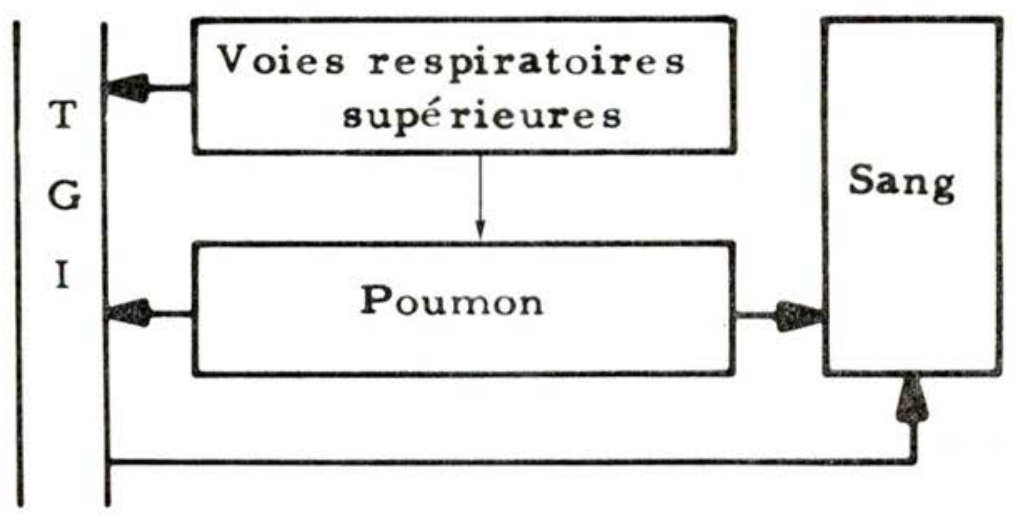

Fig. I. - Modèle de dépôt dans l'arbre respiratoire adopté par la C.I.P.R. en 1959.

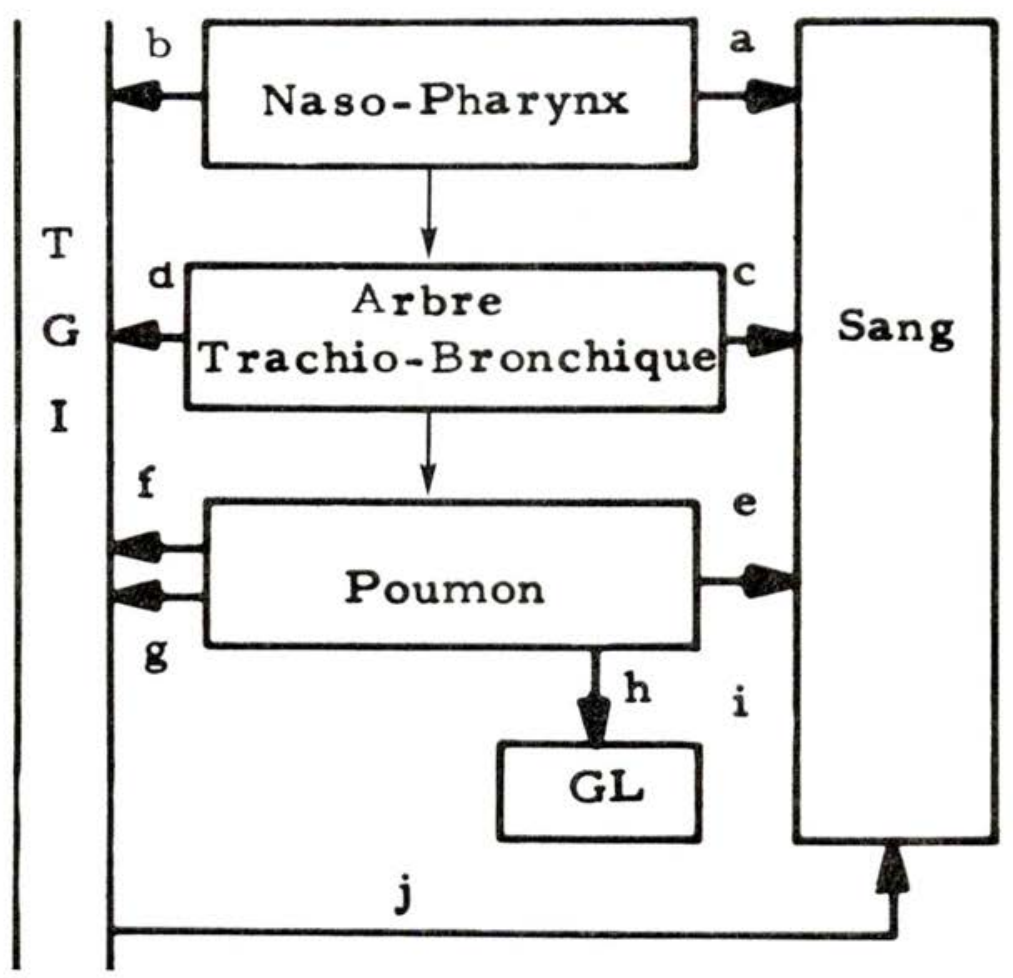

FiG. 2. - Modèle de dépôt proposé par le Groupe de Travail de la C.I.P.R. en 1966. 


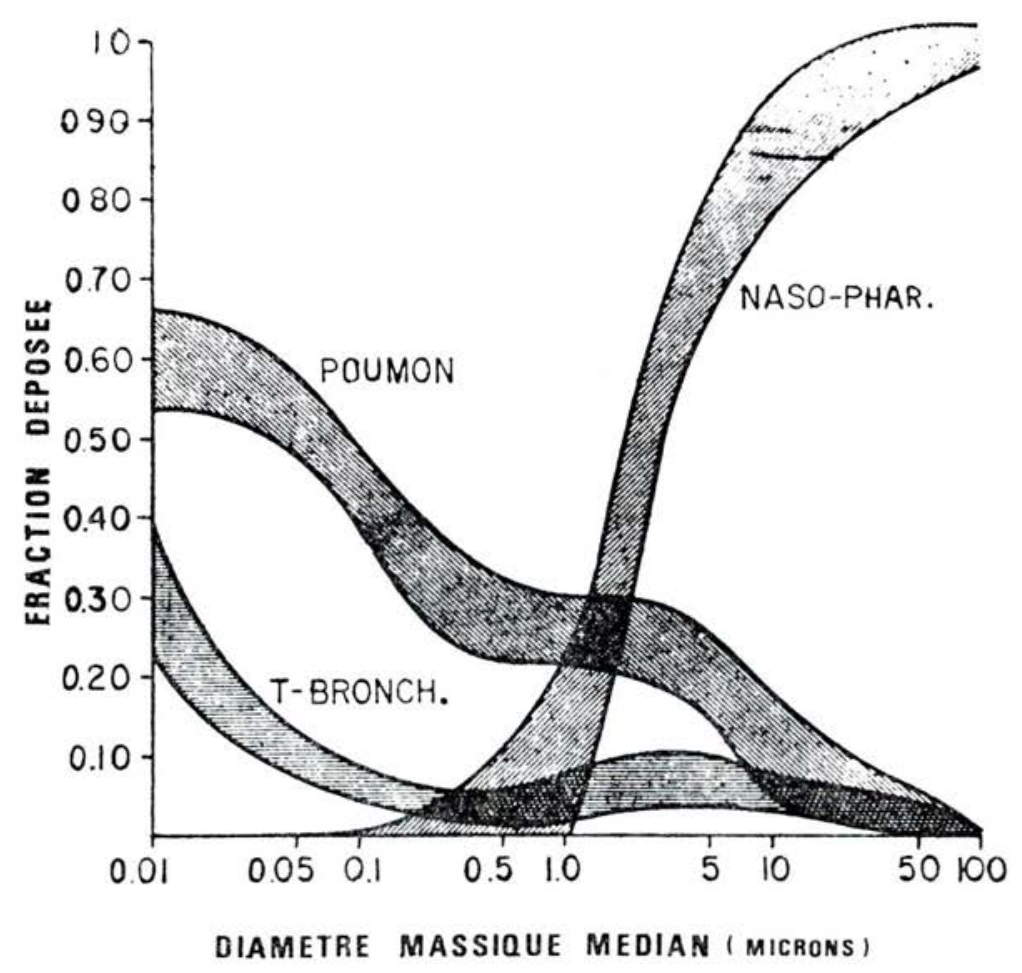

FIg. 3. - Dépôt des particules dans l'arbre respiratoire en fonction du diamètre massique médian. 